\title{
Isotope-selective Microscale Imaging of Radioactive Cs without Isobaric Interferences Using Sputtered Neutral Mass Spectrometry with Two-step Resonant Ionization Employing Newly-developed Ti:Sapphire Lasers
}

\author{
Tetsuo SaKamoto, ${ }^{* 1 \dagger}$ Masato Morita, ${ }^{* 1}$ Keita KAnENARI, ${ }^{* 1}$ Hideki Tomita, ${ }^{* 2}$ Volker SonNensCheIn, $* 2$ \\ Kosuke SAITO, $* 2$ Masaya OHASHI, $* 2$ Kotaro KATO, $* 2$ Tetsuo IGUCHI, $* 2$ Toshihide KAWAI, $* 3$ \\ Takeo OKUMURA, ${ }^{* 3}$ Yukihiko SATOU, ${ }^{* 4}$ and Ikuo WAKAIDA $* 4$ \\ *1 Department of Applied Physics, Kogakuin University, 2665-1 Nakano-machi, Hachioji, Tokyo 192-0015, Japan \\ *2 Department of Energy Engineering, Nagoya University, Furo-cho, Chikusa, Nagoya 464-8603, Japan \\ *3 Japan Neutron Optics Inc., 20-5 Takeshima-cho, Gamagori, Aichi 443-0031, Japan \\ *4 Collaborative Laboratories for Advanced Decommissioning Sciences, Japan Atomic Energy Agency, 790-1 \\ Otsuka, Motooka, Tomioka, Fukushima 979-1151, Japan
}

\begin{abstract}
The characterization of radionuclides in Fukushima is important to determine their origins and current state in the environment. Radionuclides exist as fine particles and are mixed with other constituents. A measurement method with both micro-imaging capability and highly selective element detection is necessary to analyze these particles. We developed such an imaging technique using a time-of-flight secondary ion mass spectrometry and wavelength-tunable Ti:Sapphire lasers for the resonance ionization of target elements without mass interference. This is called resonant laser ionization sputtered neutral mass spectrometry. The instrument has a high lateral resolution and a higher ionization selectivity using two-step resonance excitation of $\mathrm{Cs}$ with two lasers at different wavelengths. Optimization of the wavelength for resonance ionization using a Cs compound was performed, and a real environmental particle containing radioactive Cs was analyzed. Isotope images of three kinds of Cs were successfully obtained without interfere from Ba isotopes for the first time.
\end{abstract}

Keywords TOF-SIMS, resonance ionization, SNMS, surface micro imaging, radioactive cesium, radionuclides

(Received May 30, 2018; Accepted June 25, 2018; Advance Publication Released Online by J-STAGE July 6, 2018)

\section{Introduction}

After the nuclear power station accident at Fukushima in 2011, a variety of radionuclides were released into the environment as fine particles. ${ }^{1}$ These particles fell out and were mixed with radioactive materials originating from past nuclear bomb experiments. Smaller particles called aerosols drift in the air for a long time and are easily raised into the atmosphere. Therefore, it is of concern that aerosols containing radionuclides still migrate within the environment. Therefore, to clarify the actual state of radioactive particles from the Fukushima accident and their dynamic state in the environment, a new micro-imaging technique that can analyze radioactive elements and the isotope ratio of radionuclides for small particles is required.

Radioactive Cs has a large amount of emission, and absorption into plants is of great concern. Various analysis techniques have been employed to visualize the distribution of Cs in plants. ${ }^{2}$ Another concern is particulate matter containing Cs, such as atmospheric aerosols and soils. The distribution of $\mathrm{Cs}$ in small

$\dagger$ To whom correspondence should be addressed.

E-mail: ct13087@ns.kogakuin.ac.jp particles has been analyzed using electron microscopic analytical methods, such as SEM-EDX. ${ }^{3}$ However, EDX (characteristic $\mathrm{X}$-ray detection) cannot separate isotopes, and the isotope ratio of radionuclides is very important for determining their origins and histories. ${ }^{4}$ Mass spectrometry is a unique method used for analyzing isotopes by separating their mass-to-charge ratio $\mathrm{m} / \mathrm{z}$. Secondary ion mass spectrometry (SIMS) is an established surface imaging technique and is generally suitable for trace analysis in the micro-scale due to its low detection limits. In particular, time-of-flight SIMS (TOF-SIMS) allows the determination of mass spectra without $\mathrm{m} / \mathrm{z}$, scan, and has a high transmission. Therefore, it is suitable for the analysis of multiple elements and unknown samples. Generally, SIMS is effective for analyzing solid samples and has demonstrated high sensitivity on radioactive fine particles using conventional SIMS. ${ }^{5}$ However, it has a serious problem in that isobaric interference due to nonselective ionization with ion beam sputtering occurs in SIMS. ${ }^{6}$ To overcome this problem, resonance ionization mass spectrometry (RIMS) is known as an element-selective analysis for the detection of radionuclides. ${ }^{7,8}$

Wavelength-tunable laser(s) are used in RIMS. If an experimenter wishes to analyze a specific element, the wavelength is adjusted precisely to the electronic excitation 


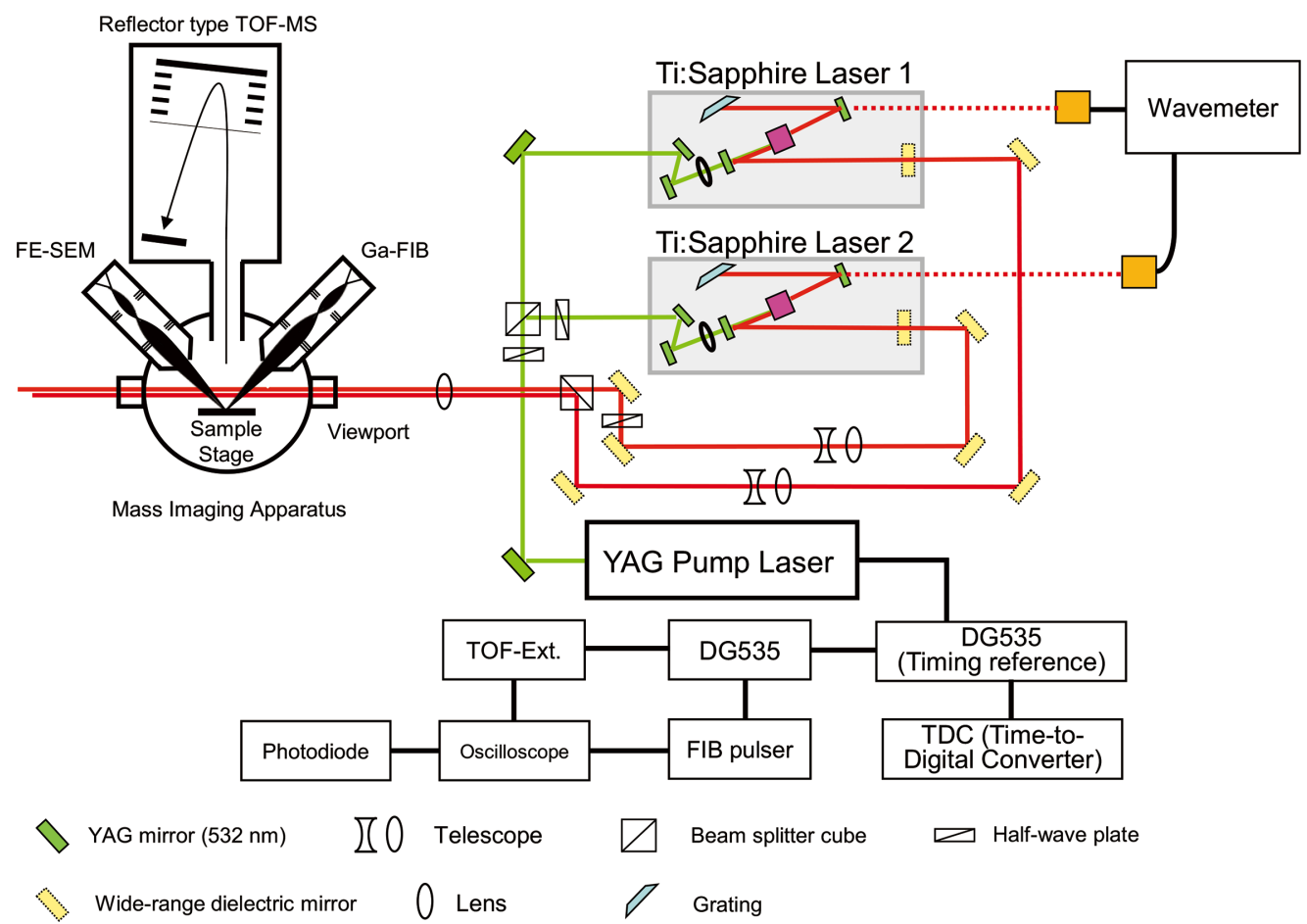

Fig. 1 Experimental setup of the two-step resonant ionization SNMS imaging system using two Ti:Sapphire lasers.

level of the element. Since the photon energy and the energy level in the atom are matched, resonance absorption occurs, and subsequent absorption of another photon results in ionization. RIMS is a technique with high selectivity as the atomic energy levels depend on the kind of element. This selectivity overcomes the problem of isobaric interference, which is a main problem in SIMS.

On the basis of this background, we are currently developing a resonant laser ionization sputtered neutral mass spectrometry (R-SNMS) technique. To improve ionization selectivity, lasers with two different wavelengths are used simultaneously. The lasers specifications are identical and have been newly designed for use in R-SNMS.

In this paper, we report on the current state of development and an example application to a real small sample containing radioactive Cs to confirm its ability to image radioisotopes in a microscopic field of view.

\section{Experimental}

\section{Resonant ionization samples}

As a model sample, anhydrous $\mathrm{Cs}_{2} \mathrm{CO}_{3}$ (Wako Chemicals, purity $>95.0 \%$ ) was used to evaluate the isotopic content of Cs using R-SNMS. The powder was lightly pressed onto a smooth indium plate (Nilaco, purity $99.99 \%$ ) and mounted on the sample stage in the R-SNMS apparatus.

A small particle containing radioactive Cs was used to ensure the usefulness of the R-SNMS technique. The particle was retrieved from soil collected $2 \mathrm{~km}$ away from Fukushima Daiichi Nuclear Power Station. The diameter of the particle was about 100 to $150 \mu \mathrm{m}$. The Cs-containing particle was found with radiography among a number of other kinds of soil. It has been said that Cs-containing particles are categorized into two types of particles, called Type A and Type $B{ }^{8}$ Type $A$ is
$2-10 \mu \mathrm{m}$ in diameter and contains Cs with high concentration. Type B is a coarse particle compared with Type A, and the diameter is in the sub-mm range. The cesium concentration in Type B is much lower than that in Type A. For the first attempt in this study, a particle (Type B) was fixed on the sample stage with electroconductive carbon tape.

\section{Isotope imaging apparatus}

Figure 1 shows the experimental setup of the R-SNMS apparatus. It consists of a TOF-SIMS apparatus and two tunable Ti:Sapphire lasers that are used for resonant ionization. The TOF-SIMS system used here was developed by us, and was already equipped with a non-resonant laser (NR-SNMS) measurement system ${ }^{9}$ using a 266-nm wavelength laser (fourth harmonic of an Nd:YAG laser). NR-SNMS at $266 \mathrm{~nm}$ was developed as a method to enhance the sensitivity of aromatic organics compared with SIMS. ${ }^{10}$ Since a UV pulsed laser is used in NR-SNMS, it is possible to ionize sputtered neutral particles via simultaneous two photon absorption if the sum of the energy of the two photons is greater than the ionization potential of the atoms or molecules being analyzed. In this nonresonant ionization scheme, there is no elemental selectivity; therefore, isobaric interference occurs frequently. The most severe isobaric interference is ${ }^{137} \mathrm{Cs}$ and ${ }^{137} \mathrm{Ba}$. Their exact mass are $136.907 \mathrm{amu}$ and $136.905822 \mathrm{amu}$, respectively. If one try to separate them by only the very small mass difference, mass resolution $\mathrm{m} / \Delta m$ greater than $10^{5}$ is necessary. In practice, the amount of ${ }^{137} \mathrm{Ba}$ is larger than ${ }^{137} \mathrm{Cs}$ in environmental samples, therefore, much higher mass resolution is required, and is unrealistic.

To avoid isobaric interference in this study, Cs neutrals sputtered from solid surfaces by ion beam irradiation were ionized using a three-photon resonant ionization process with photons of two distinct energies. Figure 2 shows the resonant ionization scheme for Cs used in this study. There are many 


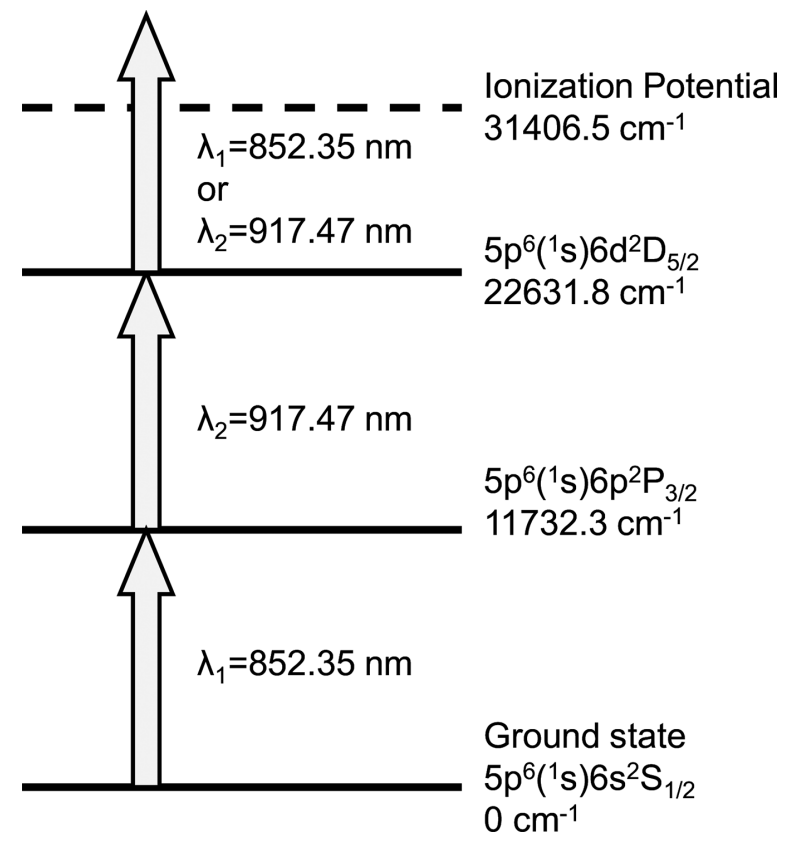

Fig. 2 Two-color and three-photon resonant ionization scheme of Cs.

alternative ionization schemes via different excitation levels. This ionization scheme used here was carefully selected under the following considerations. First, both $\lambda_{1}$ and $\lambda_{2}$ were within the tuning range of a Ti:Sapphire laser $(680$ to $960 \mathrm{~nm}$ and its second harmonic). Second, the rather long lifetimes of each intermediate excited state (around $100 \mathrm{~ns}$ ) were chosen, so that they would be sufficiently long compared with the stability of the pulse timing difference between $\lambda_{1}$ and $\lambda_{2}{ }^{11}$ Two identical Ti:Sapphire lasers were developed by us based on a conventional Ti:Sapphire laser. ${ }^{12}$ The lasers have a high repetition rate (up to $10 \mathrm{kHz}$ ), continuous wide tuning range by using a diffraction grating instead of etalons for wavelength selection, and high pulse energy $(170 \mu \mathrm{J} /$ pulse at $800 \mathrm{~nm}){ }^{13}$ A commercial Nd:YAG laser (Photonics Industries International Inc., DM60532) with a repetition rate of $10 \mathrm{kHz}$ at $532 \mathrm{~nm}$ was used as a pump laser for the Ti:Sapphire lasers after the output beam was split using a polarizing beam splitter cube (see Fig. 1). The laser wavelength was measured with a wavemeter (HighFinesse Inc., WS-6) in real-time. The linewidth of the laser was 10 to $30 \mathrm{pm}$.

The TOF-SIMS apparatus is equipped with a focused ion beam (FIB) with a minimum beam diameter of $40 \mathrm{~nm}$ in the pulsed mode. The FIB was run at an acceleration voltage of $30 \mathrm{kV}$ and included a thermal field emission electron beam for SEM observation, a main vacuum chamber with quartz glass laser windows, and a reflector-type TOF mass spectrometer. The base pressure inside the main chamber was around $5 \times$ $10^{-6} \mathrm{~Pa}$. The FIB was pulsed by a chopping plate inside the FIB column as is typically done in TOF-SIMS or TOF-NR-SNMS. In the case of two-color R-SNMS, two lasers are required to maintain precise synchronization within the FIB. As shown in Fig. 1, two digital delay generators (Stanford Research Systems Inc., DG535) were used for timing adjustments. The first DG535 was operated with an internal trigger mode at $10 \mathrm{kHz}$, and the timing of the first DG535 was used as the reference for the other delayed triggers. The pump laser was operated with the external trigger from the first DG535, which included an appropriate delay time, since the actual laser pulse is generated after the trigger signal.
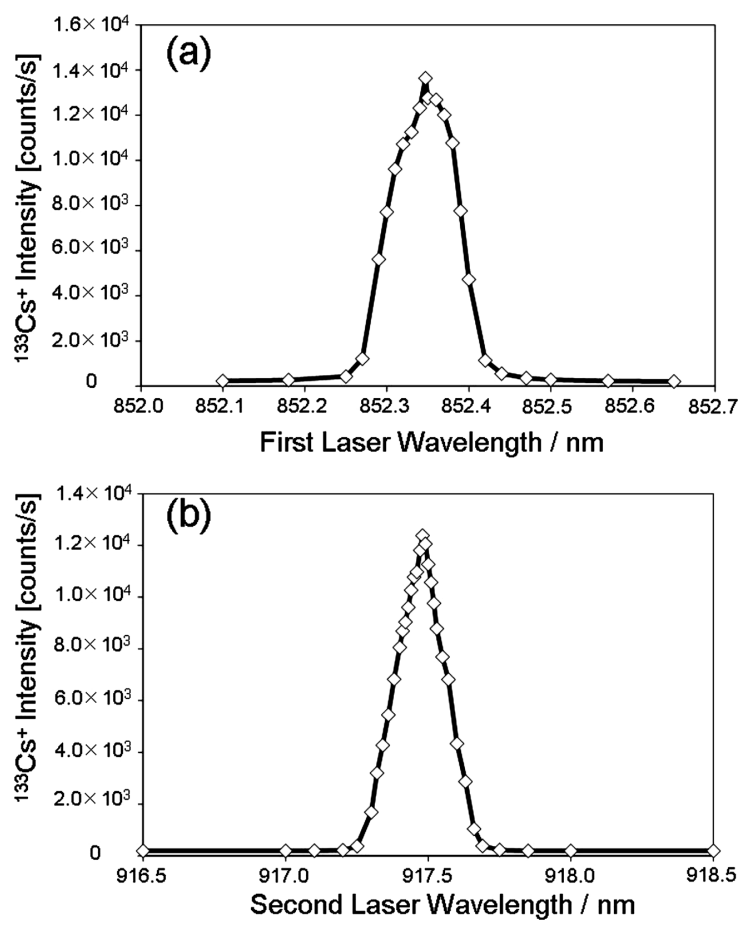

Fig. $3{ }^{133} \mathrm{Cs}^{+}$count rate as a function of the laser wavelength. (a) $1 \mathrm{st}$ step resonance and (b) 2nd step resonance.

\section{Results and Discussion}

Evaluation of element-selective ionization in R-SNMS using cesium reagent

The resonant ionization spectrum for $\mathrm{Cs}$ was measured in R-SNMS using the scheme shown in Fig. 2. The resonance measurement was carried out at each excitation wavelength. While the precise wavelengths required for the 1st and 2nd excitation steps were searched by the authors based on an atomic line data base, ${ }^{14}$ it was still needed to reexamine by measurement. This is due to the fact that sputtered neutral atoms have a few eV kinetic energy and nonuniform emission direction, which may result in a Doppler shift and/or broadening of the resonance wavelength. First the two lasers were set at the literature values of $\lambda_{1}=852.35$ and $\lambda_{2}=917.47 \mathrm{~nm}$ with the maximized pulse energy, respectively. After measuring the exact resonance wavelengths, the pulse energies from both lasers were reduced until the non-resonant ionization $\mathrm{Cs}^{+}$signals disappeared. As a result, the optimum laser pulse energies were 3.0 and $0.2 \mu \mathrm{J} /$ pulse for the $1 \mathrm{st}$ and 2 nd excitation steps, respectively. Figure 3 shows the measurement results. The best excitation wavelengths for the 1st step transition and 2nd step transition were determined to be 852.347 and $917.471 \mathrm{~nm}$, respectively.

Figure 4 shows microscopic images of a $\mathrm{Cs}_{2} \mathrm{CO}_{3}$ reagent particle acquired with R-SNMS using the ionization scheme shown in Fig. 2 and the optimized wavelengths mentioned above. This confirms the imaging capability of R-SNMS, as well as conventional TOF-SIMS. The time required for the imaging was $7 \mathrm{~min}$, which is identical to that of TOF-SIMS, because the laser pulse repetition rate is high $(10 \mathrm{kHz})$.

Isotopic micro-imaging of a real environmental particle

Figure 5 shows an FIB-induced secondary electron image of 
Type B Cs-containing particle collected $2 \mathrm{~km}$ away from Fukushima Dai-ichi Nuclear Power Station. Prior to the R-SNMS analysis, it was confirmed that this particle contained radioactive $\mathrm{Cs}$ using radiography. The particle had an uneven shape, and very fine particles on the surface. Next, a R-SNMS measurement of the entire particle was performed within the same field of view, and the radioactive Cs was analyzed. The result of this analysis (not shown as a figure) revealed that radioactive Cs existed on only a portion of this particle. Next, both laser wavelengths were optimized again, since the Doppler shift and broadening were thought to be different from those in the small particle discussed in the previous section. The laser axis was just above and parallel to the sample carrier surface, while the incident angle of the FIB was at 45 degrees with respect to the sample carrier. Therefore, in the case of analysis for a large steric sample like this particle, the FIB irradiation side is shaded from laser irradiation. The angular distribution of sputtered atoms does not strongly depend on the FIB incidence angle, but has the peak intensity in the direction of the sample
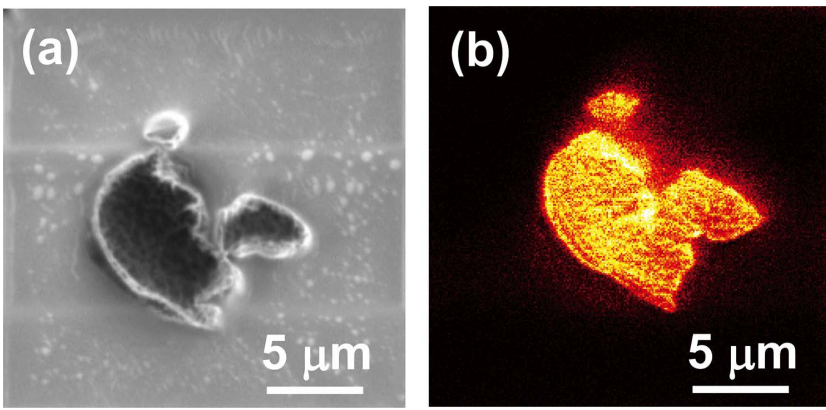

Fig. 4 (a) FIB-induced secondary electron image of a $\mathrm{Cs}_{2} \mathrm{CO}_{3}$ particle on an In plate. (b) Two-color R-SNMS image of ${ }^{133} \mathrm{Cs}$. surface normal. Therefore, the sputtered neutrals from the shaded side have a rather large velocity component with respect to the laser irradiation axis. Because of this, a noticeable Doppler shift should occur. Considering these situations, the resonant wavelength shifts toward a longer wavelength. In fact, the first excitation wavelength was optimized to $852.447 \mathrm{~nm}$ from $852.347 \mathrm{~nm}$ (which is the best value in the case of a flat sample, as shown in Fig. 3) and the second was optimized to $917.580 \mathrm{~nm}$ from $917.471 \mathrm{~nm}$.

Figure 6 shows the R-SNMS imaging results of the particle. The square area shown in Fig. 6(a) was imaged. Figures 6(b) and 6(c) were acquired without laser ionization, i.e., in SIMS

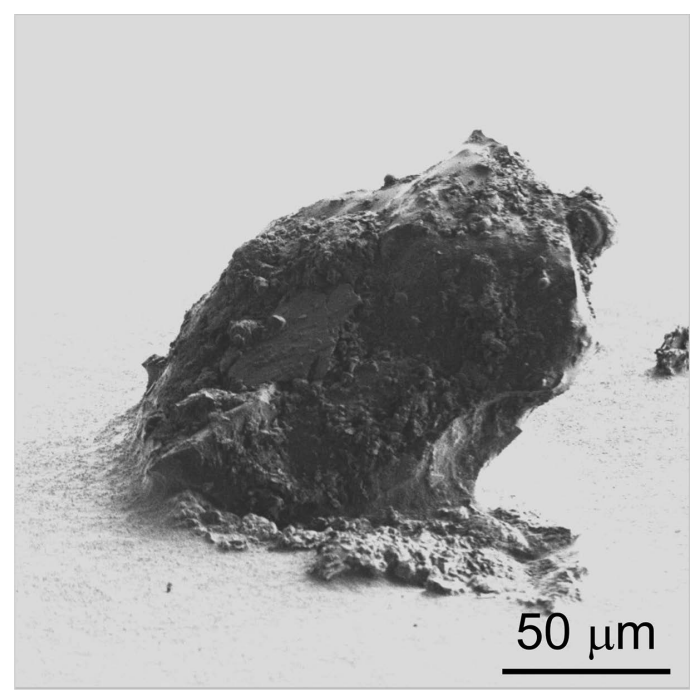

Fig. 5 FIB-induced secondary electron image of a radioactive Cs-containing particle (Type B).
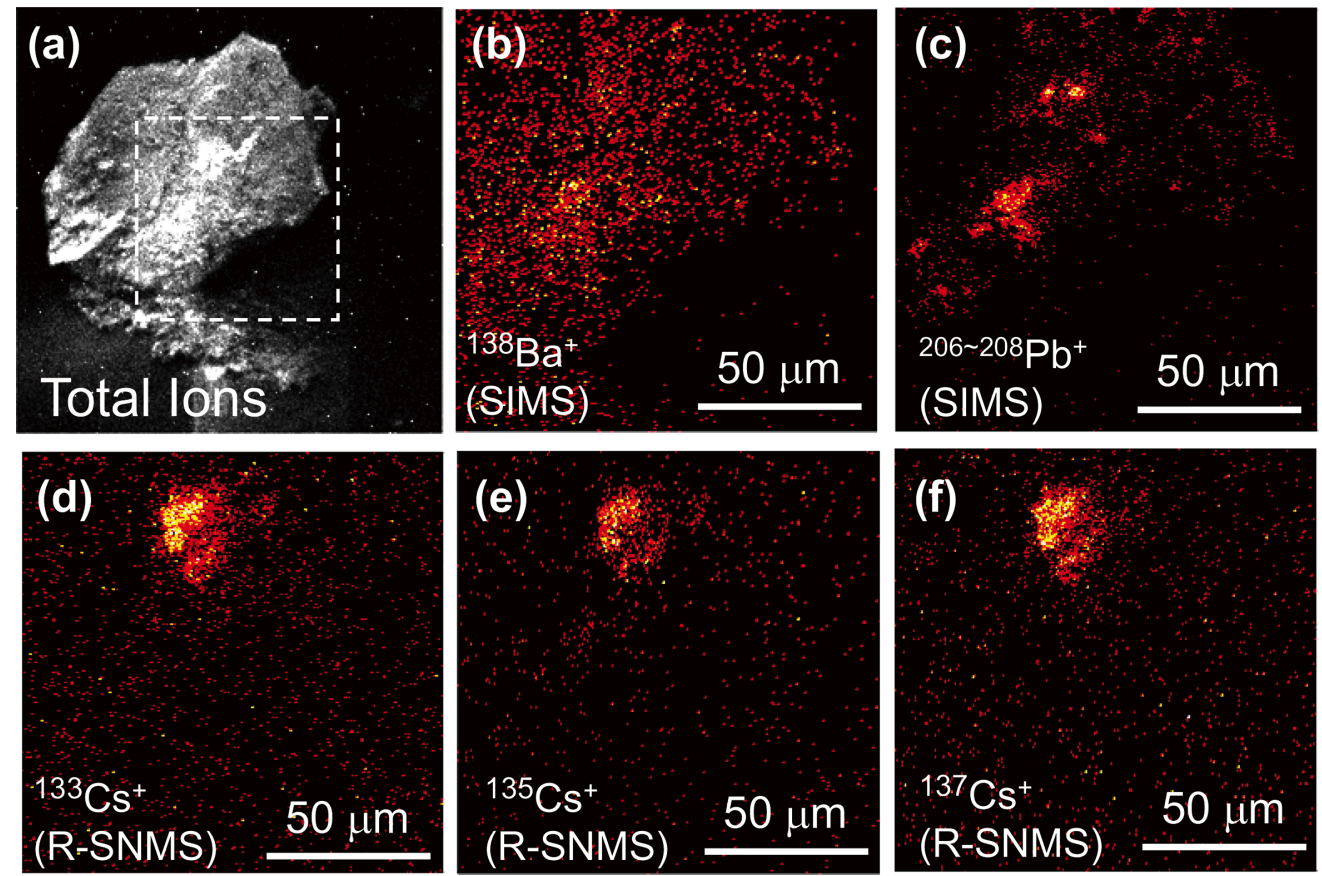

Fig. 6 (a) FIB-induced total ion image of the particle. (b) TOF-SIMS images of ${ }^{138} \mathrm{Ba}^{+}$and (c) ${ }^{206-208} \mathrm{~Pb}^{+}$. Panels (d) to (f) show two-color R-SNMS images of Cs isotopes. 
Table 1 Cs isotope ratio measured by R-SNMS from the Cscontaining particle and theoretical ratios in nuclear reactor Unit 1 to 3

\begin{tabular}{lcclll}
\hline & $\begin{array}{c}\text { Detected } \\
\text { raw counts }\end{array}$ & $\begin{array}{c}\text { Isotope ratio } \\
\left({ }^{137} \mathrm{Cs}=1\right)\end{array}$ & Unit 1 & Unit 2 & Unit 3 \\
\hline${ }^{133} \mathrm{Cs}$ & 601 & $1.11 \pm 0.00^{\mathrm{b}}$ & 1.02 & 0.990 & 0.992 \\
${ }^{134} \mathrm{Cs}$ & 0 & 0 & 0.065 & 0.074 & 0.072 \\
${ }^{135} \mathrm{Cs}$ & 190 & $0.351 \pm 0.01^{\mathrm{b}}$ & 0.396 & 0.341 & 0.350 \\
${ }^{137} \mathrm{Cs}$ & 461 & 1 & 1 & 1 & 1 \\
\hline
\end{tabular}

a. The decay after the incident was considered based on its half-life for ${ }^{135} \mathrm{Cs}$ and ${ }^{137} \mathrm{Cs}$.

b. Statistical errors were estimated by square root of the counts.

mode. ${ }^{138} \mathrm{Ba}^{+}$ions were distributed over the entire area of the particle. $\mathrm{Pb}$ was also detected, but the distribution was inhomogeneous, and small particles seemed to occupy the surface. R-SNMS imaging was then performed in the same area using the optimized laser wavelengths mentioned above. The time required for mapping was $20 \mathrm{~min}$, and the FIB current was set at $4 \mathrm{nA}$ (measured in DC mode). Figures 6(d), 6(e), and 6(f) are ${ }^{133} \mathrm{Cs}^{+},{ }^{135} \mathrm{Cs}^{+}$, and ${ }^{137} \mathrm{Cs}^{+}$images, respectively. First, it is clear that when $m / z=137$, the image (Fig. 6(f)) is completely different from the ${ }^{138} \mathrm{Ba}^{+}$image (Fig. 6(b)). This means that the $m / z=137$ image certainly corresponds to ${ }^{137} \mathrm{Cs}^{+}$, because ${ }^{137} \mathrm{Ba}$ isotope has an $11 \%$ natural abundance. If selective ionization of ${ }^{137} \mathrm{Cs}^{+}$was not successful, Fig. 6(f) should resemble Fig. 6(b). The selective detection of Cs was further confirmed because all three Cs isotopes were imaged at the same position and with closely identical form. From these R-SNMS images, it was shown that $\mathrm{Cs}$ existed in the shape of a particle with a diameter of around $30 \mu \mathrm{m}$ on the surface of the larger main particle. This is the first demonstration of the successful visualization of radioactive $\mathrm{Cs}$ isotopes in a microscopic field of view. In the case of $\mathrm{Cs}_{2} \mathrm{CO}_{3}$ reagent (Fig. 4), the useful yield (number of detected target ions/sputtered target ions) of ${ }^{133} \mathrm{Cs}^{+}$ion was calculated to be $5.27 \times 10^{-3}$ using the value of the $\mathrm{Ga}^{+}$FIB dose and the detected number of ${ }^{133} \mathrm{Cs}^{+}$ions. Using this useful yield, the number of ${ }^{137} \mathrm{Cs}$ atoms on the surface in the imaging area was estimated to be $8.75 \times 10^{4}$. This value corresponds to $3.38 \times 10^{-7} \mathrm{~Bq}$. Since Cs was not uniformly distributed in the particle, the surface atomic concentration of ${ }^{137} \mathrm{Cs}$ was estimated for the imaged Cs-concentrated spot by assuming that the surface matrix was $\mathrm{SiO}_{2}$. The result was $10.9 \mathrm{ppb}$ for ${ }^{137} \mathrm{Cs}$.

The isotope ratio of $\mathrm{Cs}$ was discussed by comparing the measured values with the simulated ones. ${ }^{15}$ Table 1 summarizes the results. First, the detected raw count for each isotope was extracted from the corresponding isotope images. ${ }^{134} \mathrm{Cs}$ was not detected because its half-life is short (about 2 years), and its concentration was originally low at the time of the incident. ${ }^{15}$ The isotope ratio was defined by setting the ${ }^{137} \mathrm{Cs}^{+}$counts to unity. Note that ${ }^{135} \mathrm{Cs}$ has very long half-life $\left(2.3 \times 10^{6}\right.$ years $)$. This is called a difficult-to-analyze nuclide because the probability of $\gamma$-ray emission is very small. It can be detected only with the method presented here. Since the R-SNMS analysis was performed in November 2017, the isotope ratio was calculated by considering the elementary nuclear decay of each isotope after the incident based on its half-life, i.e., the isotope ratio corresponds to that at the time of the incident in March 2011. The right-three columns list the theoretically calculated isotope ratios, considering the state of each nuclear reactor. One can see that the isotope ratio obtained using R-SNMS is consistent with the theoretical ratios. In this analysis, one cannot specify which reactor originally produced the particle. This is because the theoretical isotope ratios were calculated by assuming a homogeneous isotope ratio in the reactor. However, it was confirmed that the particle surely originated from the Fukushima Dai-ichi nuclear power station.

\section{Conclusions}

In order to acquire images of radioactive Cs isotopes, we developed a two-step excitation R-SNMS by using two Ti:Sapphire lasers with an existing TOF-SIMS apparatus. First, the appropriate laser wavelengths for selective ionization were confirmed using a model sample of the $\mathrm{Cs}_{2} \mathrm{CO}_{3}$ reagent. The resonant ionization spectrum of each excitation step and the R-SNMS image were successfully obtained from a microscopic area. We then attempted to analyze a Cs-containing particle sample (called Type B) collected near Fukushima Dai-ichi nuclear power station. After optimizing the laser wavelengths to compensate for the Doppler shift, the Type B particle was analyzed using both TOF-SIMS and R-SNMS modes. From the R-SNMS analysis, it was found that radioactive Cs existed as small particulate matter on the particle body. Furthermore, the isotope ratio obtained using R-SNMS was consistent with the theoretical value by including the difficult-to-analyze ${ }^{135} \mathrm{Cs}$ nuclide. This R-SNMS method is a new tool for investigating the current state of radionuclides existing as small particles in air, soil, or plants. Therefore, we can conclude that the two step R-SNMS method will give us detailed information for human health, decontamination of radionuclides, and decommissioning of the reactors.

\section{Acknowledgements}

This study supported by "Development of System and Technology for Advanced Measurement and Analysis" SENTAN, JST.

\section{References}

1. K. Adachi, M. Kajino, Y. Zaizen, and Y. Igarashi, Sci. Rep., 2013, 3, 2554.

2. Y. Noda, J. Furukawa, T. Aohara, N. Nihei, A. Hirose, K. Tanoi, T. M. Nakanishi, and S. Satoh, Sci. Rep., 2016, 6, 38360.

3. J. Imoto, A. Ochiai, G. Furuki, M. Suetake, R. Ikehara, K. Horie, M. Takehara, S. Yamasaki, K. Nanba, T. Ohnuki, G. T. W. Law, B. Grambow, R. C. Ewing, and S. Utsunomiya, Sci. Rep., 2017, 7, 5409.

4. S. A. Hodgson, G. J. Ham, M. J. Youngman, G. Etherington. and G. N. Stradling, J. Radiol. Prot., 2004, 24, 369.

5. Y. Ranebo, P. M. L. Hedberg, M. J. Whitehouse,c K. Ingenerid, and S. Littmanna, J. Anal. At. Spectrom., 2009, 24, 277.

6. G. Tamborini and M. Betti, Mikrochim. Acta, 2000, 132, 411.

7. M. Miyabe, M. Oba, M. Kato, I. Wakaida, and K. Watanabe, J. Nucl. Sci. Technol., 2006, 43, 305.

8. Y. Satou, K. Sueki, K. Sasa, H. Yoshikawa, S. Nakama, H. Minowa, Y. Abe, I. Nakai, T. Ono, K. Adachi, and Y. Igarashi, Geochem. J., 2018, 52, 137.

9. T. Sakamoto, M. Koizumi, J. Kawasaki, and J. Yamaguchi, Appl. Surf. Sci., 2008, 255, 1617. 
10. K. Ohishi, T. Sakamoto, J. Saikawa, N. Ishigaki, K. Tojo, Y. Ido, S. Hayashi, S. Ishiuchi, K. Misawa, and M. Fujii, Anal. Sci., 2013, 29, 291.

11. T. L. Correll, V. Horvatic, N. Omenetto, and C. Vadla, Spectrochim. Acta, Part B, 2005, 60, 765 .

12. S. Rothe, B. A. Marsh, C. Mattolat, V. N. Fedosseev, and K. Wendt, J. Phys.: Conf. Ser., 2011, 312, 052020.

13. H. Tomita, A. Nakamura, D. Matsui, R. Ohtake, V. Sonnenschein, K. Saito, K. Kato, M. Ohashi, V. Degner, K.
Wendt, M. Morita, T. Sakamoto, T. Kawai, T. Okumura, I. Moore, and T. Iguchi, Progress in Nuclear Science and Technology, accepted for publication.

14. R. L. Kurucz and B. Bell, Cambridge, Mass.: Smithsonian Astrophysical Observatory. Atomic Line Data Kurucz CDROM, 1995, 23.

15. K. Nishihara, H. Iwamoto, and K. Suyama, JAEA-Data/ Code, 2012, 018, 13. 